\title{
章 \\ Demodiciose canina: estudo retrospectivo dos casos atendidos no Hospital Veterinário da Universidade Federal Rural do Rio de Janeiro (2013-2017)
}

\author{
[Canine demodicosis: a retrospective study of cases from the Federal Rural University of Rio de \\ Janeiro Veterinary Hospital (2013-2017)]
}

\section{"Artigo Científico/Scientific Article"}

\author{
Natália Lôres Lopes ${ }^{1 *}$, Marília Alves Machado ${ }^{1}$, Dayane Caicó Colares Araújo ${ }^{1}$, \\ Carolina de Moraes Barbalho², Bruna Land Manier ${ }^{2}$, Fabrícia Ferreira e Ferreira ${ }^{3}$, \\ Thiago Souza Costa ${ }^{1}$, Julio Israel Fernandes ${ }^{4}$
}

\begin{abstract}
${ }^{1}$ Programa de Pós-graduação em Medicina Veterinária, Universidade Federal Rural do Rio de Janeiro, Seropédica-RJ, Brasil.

${ }^{2}$ Setor de Dermatologia, Hospital Universitário, Universidade Federal Rural do Rio de Janeiro, Seropédica-RJ, Brasil. ${ }^{3}$ Médica Veterinária Autônoma.

${ }^{4}$ Departamento de Medicina e Cirurgia Veterinária, Universidade Federal Rural do Rio de Janeiro, Seropédica-RJ, Brasil. *Autor para correspondência/Corresponding author: E-mail: natloresvet@gmail.com
\end{abstract}

\section{Resumo}

A demodiciose é uma das principais dermatopatias parasitárias atendidas na rotina de clínicas veterinárias, acometendo principalmente animais jovens, cujo diagnóstico pode ser realizado através do raspado cutâneo profundo, tricograma e fita de acetato. Os sinais dermatológicos, incluindo alopecia, eritema, crostas e pápulas localizam-se principalmente na face. O objetivo do trabalho foi realizar levantamento de casos de demodiciose canina diagnosticados no setor de Dermatologia do Hospital Veterinário da Universidade Federal Rural do Rio de Janeiro no período de janeiro de 2013 a abril de 2017. Foram utilizadas 125 fichas com diagnóstico de demodiciose de um total de 1282 animais atendidos. Foi encontrada uma ocorrência de 9,75\%(125/1282) da doença em cães, predominantemente jovens, com idade inferior a um ano e sem predileção sexual. Com relação a distribuição racial, a de maior prevalência foi a sem raça definida $(29,6 \% ; 37 / 125)$, tendo como principal sinal clínico a hipotricose $(60,8 \% ; 76 / 125)$ e apresentando-se de forma generalizadas $(76,8 \% ; 96 / 125)$. O método diagnóstico mais utilizado foi o raspado cutâneo $(61,6 \% ; 77 / 125)$. Foi possível concluir que a demodiciose é uma doença que deve estar dentre os diagnósticos diferenciais na clínica de animais de companhia, principalmente em animais jovens, com idade inferior a 1 ano de idade. E que esta doença acomete principalmente região de cabeça com lesões mais frequentes de hipotricose e eritema.

Palavras-chave: dermatopatia; cães; Demodex canis.

\section{Abstract}

Demodicosis is one of the main parasitic diseases in the routine of veterinary clinics, mainly affecting young animals, whose diagnosis can be made by a trichogram, acetate tape, and skin scrapings. Dermatological signs, including alopecia, erythema, crusts, and papules are mainly on the face. The aim of this study was to perform a survey of canine demodicosis cases diagnosed at the Dermatology Department of the Veterinary Hospital of the Federal Rural University of Rio de Janeiro from January 2013 to April 2017. One hundred and twenty-five from a total of 1282 medical records from dogs diagnosed with demodicosis were used. An occurrence of 9.75\% (125/1282) of the disease was found in dogs, predominantly young, aged less than one year, and without sexual preference. The most prevalent breed was the mixed breed $(29.6 \% ; 37 / 125)$, with hypotrichosis as the main clinical sign $(60.8 \% ; 76 / 125)$ and presenting as the generalized form $(76.8 \%$; 96/125). The most used diagnostic method was skin scraping $(61.6 \% ; 77 / 125)$. It can be concluded that demodicosis must be among the differential diagnoses, especially in young animals with less than 1 year of age and that this disease mainly affects areas in the head, and hypotrichosis and erythema are the most frequent lesions observed.
\end{abstract}

Keywords: dermatopathy; dogs; Demodex canis. 


\section{Introdução}

A demodiciose canina é caracterizada pela presença em número maior que o normal de ácaros do gênero Demodex (Miller et al., 2013), residente principalmente nos folículos pilosos e glândulas sebáceas do cão (Miller et al., 2013; Delayte, 2016). Em um estudo recente foi possível amplificar o DNA do ácaro da pele da maioria dos cães saudáveis analisados através da técnica de PCR demonstrando que são habitantes normais em baixa quantidade da pele de cães (Ravera et al., 2013). Demodex canis é a espécie mais relatada em cães, porém, Demodex injai e um ácaro ainda inominado chamado não oficialmente de Demodex cornei, já foram descritos (Miller et al., 2013; Sivajothi, 2015; Delayte, 2016).

Considerada a dermatopatia mais prevalente dentre as de etiologia parasitária (Delayte, 2016), a demodiciose canina tem ocorrência descrita na literatura brasileira variando entre $6,6 \%$ a $20,9 \%$ (Barbosa, 2006; Rocha et al., 2008; Silva et al., 2011; Gasparetto et al., 2013), podendo chegar a $40 \%$ das ectoparasitoses atendidas em hospital veterinário (Delayte et al., 2016). A alta ocorrência no Brasil contrasta com os $0,37 \%$ reportado por Bownden et al. (2017), em um hospital veterinário da Califórnia.

Segundo a literatura, a demodiciose canina é mais comum em cães jovens (Pereira et al., 2012; Pereira et al., 2015), animais de pelo curto (Pereira et al., 2012) e em cães de raça pura (Pereira et al., 2012; Pereira et al., 2015), enquanto que nos sem raça definida ocorre entre 10 a 12\% (Rocha et al., 2008; Pereira et al., 2012). Pereira et al. (2015) não observaram predisposição sexual, porém em um estudo foi observado frequência de $60 \%$ em fêmeas (Pereira et al., 2012).

A doença pode ser classificada em localizada, de curso benigno no qual a maioria dos casos tem resolução espontânea, ou generalizada que normalmente se inicia quando ainda filhotes (3-18 meses) (Miller et al., 2013). Na forma localizada há o envolvimento de até cinco áreas, (Delayte, 2016) enquanto que a generalizada é uma das mais severas doenças cutâneas caninas e pode ser disseminada desde o início, mas geralmente começa com múltiplas áreas da doença (Miller et al., 2013), com mais de cinco lesões localizadas, envolvimento completo de uma região corpórea ou ainda, o acometimento de duas ou mais regiões palmares ou plantares (Delayte, 2016).
De acordo com a faixa etária de ocorrência das primeiras manifestações também pode ser dividida como juvenil, quando o surgimento das lesões ocorre até 12 meses de idade e nas raças gigantes até os 18 meses, ou adulta (Delayte, 2016). A demodiciose generalizada juvenil tem prevalência descrita de $0,58 \%$ (Plant et al., 2011). $\mathrm{O}$ início adulto é definido com o desenvolvimento da doença em um adulto sem histórico anterior da doença e deve ser realizada avalição de uma doença subjacente (Mueller et al., 2012). Foram observados a presença de doenças concomitantes como neoplasia, hiperadrenocorticismo, hipotireoidismo, hepatite crônica, caquexia e infecções crônicas, assim como em fêmeas com filhotes e em animais submetidos a terapia com corticosteroides, tendo como possível explicação a imunossupressão (Holm, 2003).

Entre os sinais dermatológicos reportados em cães destacam-se: eritema, comedões, descamação, alopecia múltiplas coalescentes, pápulas, pústulas, furunculose, crostas e exsudação e, nos casos mais avançados, ulceração focal e tratos drenantes (Mueller et al., 2012). Ural et al. (2012) observaram o envolvimento principalmente das orelhas, olhos, lábios, queixo e membros.

O raspado cutâneo profundo é considerado o método de diagnóstico padrão (Saridomichelakis et al., 2007; Delayte, 2016). Outro método menos traumático e doloroso e útil em áreas difíceis de obter amostras como as patas, região interdigital, comissura labial e região periocular é a impressão em fita de acetato (Pereira et al., 2012). Também é possível diagnosticar a demodiciose através do tricograma (Miller et al., 2013) mas sua sensibilidade é relatada entre $73 \%$ (Cury et al., 2013) e $85,1 \%$ (Saridomichelakis et al., 2007).

$\mathrm{O}$ exame direto do exsudato de pústulas ou tratos drenantes podem revelar ácaros (Mueller et al., 2012) e em situações que não foram possíveis observá-los, biópsias cutâneas para exame histopatológico podem se necessárias (Mueller et al., 2012; Delayte, 2016).

O objetivo do trabalho foi realizar levantamento de casos de demodiciose canina diagnosticados no Setor de Dermatologia do Hospital Veterinário da Universidade Federal Rural do Rio de Janeiro no período de janeiro de 2013 a abril de 2017. 


\section{Material e Métodos}

Por meio de um estudo retrospectivo, foram avaliadas fichas de 125 casos de cães diagnosticados com demodiciose de um total de 1282 fichas de primeiro atendimento. Todos os casos se referiram a atendimentos realizados de janeiro de 2013 a abril de 2017 no Setor de Dermatologia do Hospital Veterinário da Universidade Federal Rural do Rio de Janeiro. Foram incluídos animais com lesões cutâneas compatíveis e a identificação de ácaros do gênero Demodex por meio do exame parasitológico de raspado cutâneo, tricograma, impressão em fita de acetato, citopatológico e/ou histopatológico. Foram compilados dados e calculada a porcentagem da casuística referentes ao sexo, idade, classificação da demodiciose (juvenil e adulta; localizada e generalizada), lesões apresentadas e sua localização, bem como a técnica utilizada para o diagnóstico

\section{Resultados}

Foram realizados durante o período de janeiro de 2013 a abril de 2017, 1282 atendimentos de primeira vez de cães. Desses atendimentos 9,75\% 125/1282) foram de demodiciose, sendo $52 \%(65 / 125)$ machos e $48 \%$ (60/125) fêmeas. A idade dos pacientes variou entre dois meses de idade a 16 anos e $44,8 \%(56 / 125)$ apresentou idade inferior a um ano de idade, $40,8 \%(51 / 125)$ entre um ano e sete anos e apenas 14,4\% (18/125) superior a sete anos. Animais sem raça definida foram os mais acometidos (29,6\%) (37/125) seguidas das raças Buldogue Francês, Poodle e Yorkshire com 8\% (10/125) cada. As demais raças diagnosticadas estão disponíveis na Tabela 1.

Tabela 1. Percentual das raças de cães diagnosticadas com demodiciose no Setor de Dermatologia do Hospital Veterinário da Universidade Federal Rural do Rio de Janeiro.

\begin{tabular}{lcc}
\hline \multicolumn{1}{c}{ Raça } & Número de animais & Percentual (\%) \\
\hline Akita & 1 & 0,8 \\
American Bully & 1 & 0,8 \\
Border Collie & 2 & 1,6 \\
Buldogue Francês & 10 & 8 \\
Buldogue Campeiro & 1 & 1,6 \\
Cane-corso & 2 & 1,6 \\
Chow-Chow & 3 & 2,4 \\
Collie & 1 & 0,8 \\
Dog Alemão & 1 & 0,8 \\
Dogo Argentino & 1 & 0,8 \\
Golden Retriever & 1 & 0,8 \\
Labrador & 5 & 4 \\
Maltês & 2 & 1,6 \\
Pator Alemão & 1 & 0,8 \\
Pastor Canadense & 1 & 0,8 \\
Pequinês & 1 & 0,8 \\
Pinscher & 6 & 4,8 \\
Pit Bull & 9 & 7,2 \\
Poodle & 10 & 8 \\
Pug & 2 & 1,6 \\
Rhodesian & 2 & 1,6 \\
Rottweiler & 1 & 0,8 \\
Shar-pei & 3 & 2,4 \\
Shih-Tzu & 4 & 3,2 \\
Sem Raça Definida & 37 & 29,6 \\
Staffordshire & 1 & 0,8 \\
Teckel & 6 & 4,8 \\
Yorkshire & 10 & 8 \\
\hline
\end{tabular}

Quanto a classificação desta dermatopatia $58,4 \%(73 / 125)$ foi juvenil e $41,6 \%(52 / 125)$ adulta e $76,8 \%$ (96/125) generalizada enquanto que
$20,8 \%(26 / 125)$ foi localizada. As lesões mais observadas foram hipotricose $(60,8 \% ; 76 / 125)$, eritema $(56,8 \%$; $71 / 125)$, crosta $(43,2 \% ; 54 / 125)$, e 
alopecia e descamação com 42,4\% (53/125) cada.

Todas as lesões observadas estão descritas na Tabela 2. Quando avaliados quanto as áreas de localização das lesões (Tabela 3), as lesões na cabeça $(36 \% ; 45 / 125)$, sendo $22,4 \%(28 / 125)$ do total na face foram as mais observadas $(30,4 \%$; $38 / 125$ ), seguido das generalizadas (todo o corpo) $(30,4 \% ; 38 / 125)$, e dorso $(25,6 \% ; 32 / 125)$.

Tabela 2. Percentual de lesões apresentadas pelos cães diagnosticados com demodiciose no Setor de Dermatologia do Hospital Veterinário da Universidade Federal Rural do Rio de Janeiro.

\begin{tabular}{lcc}
\hline \multicolumn{1}{c}{ Lesão } & Número de Animais & Percentual (\%) \\
\hline Alopecia & 53 & 42,4 \\
Celulite & 2 & 1,6 \\
Colarete & 17 & 13,6 \\
Comedos & 15 & 12 \\
Crosta & 54 & 43,2 \\
Dermatite úmida aguda & 1 & 0,8 \\
Descamação & 53 & 42,4 \\
Despigmentação & 1 & 0,8 \\
Edema & 1 & 0,8 \\
Eritema & 71 & 56,8 \\
Erosão & 4 & 3,2 \\
Escoriação & 1 & 0,8 \\
Fístula & 1 & 0,8 \\
Granuloma & 1 & 0,8 \\
Hiperpigmentação & 26 & 20,8 \\
Hiperqueratose & 34 & 27,2 \\
Hipotricose & 76 & 60,8 \\
Micro abscesso & 1 & 0,8 \\
Pápula & 48 & 38,4 \\
Pústula & 39 & 31,2 \\
Seborreia oleosa & 19 & 15,2 \\
Úlcera & 4 & 3,2 \\
Vesícula & 1 & 0,8 \\
\hline
\end{tabular}

Tabela 3. Percentual das áreas acometidas por lesões em cães diagnosticados com demodiciose no Setor de Dermatologia do Hospital Veterinário da Universidade Federal Rural do Rio de Janeiro.

\begin{tabular}{lcc}
\hline \multicolumn{1}{c}{ Localização das lesões } & Número de animais & Percentual (\%) \\
\hline Abdômen & 27 & 21,6 \\
Axila & 5 & 4 \\
Cabeça & 17 & 13,6 \\
Cauda & 6 & 4,8 \\
Cervical & 13 & 10,4 \\
Dorso & 32 & 25,6 \\
Face & 28 & 22,4 \\
Generalizada & 38 & 30,4 \\
Inguinal & 5 & 4 \\
Membro anterior & 20 & 16 \\
Membro posterior & 24 & 19,2 \\
Orelha & 4 & 3,2 \\
Patas & 20 & 16 \\
Perianal & 1 & 0,8 \\
Tórax & 9 & 7,2 \\
\hline
\end{tabular}


O exame parasitológico de raspado cutâneo foi utilizado como meio de diagnóstico em $61,6 \%$ (77/125) dos casos, a impressão em fita de acetato em 44\% (55/125), o tricograma em 21,6\% (27/125) enquanto que o exame citológico em $17,6 \%$ $(22 / 125)$ e a histopatologia em apenas $1,6 \%$ $(2 / 125)$.

\section{Discussão}

A prevalência de demodiciose encontrada neste estudo foi maior do que a descrita na região da Garça em São Paulo de 6,6\% dos casos (Barbosa, 2006), porém menor do que a observada em outros estudos (Silva et al., 2011). Em relação a dados descritos mundialmente, foi menor do que os 35\% de casos observados na Índia (Nayak et al., 1997). Os dados obtidos neste estudo através do conhecimento da casuística desta dermatopatia no estado do Rio de Janeiro foram importantes para auxiliar o clínico no diagnóstico epidemiológico mais preciso e dessa forma, favorecer o tratamento mais rápido e eficaz.

Neste trabalho não foram observadas diferença entre machos e fêmeas, assim como observado por outros autores (Nayak et al., 1997; Pereira et al., 2015), indicando que ambos os sexos podem ser igualmente susceptíveis. Entretanto Pereira et al. (2012) observaram maior ocorrência em fêmeas.

Verificou-se uma maior incidência da forma juvenil, que acomete cães até 1 ano de idade, corroborando com o fato de que o quadro clínico geralmente se inicia em cães jovens (Delayte, 2016).

Cães sem raça definida foram mais observados nesse estudo (29,6\%) assim como por Lemarie et al. (1996), com prevalência maior que a descrita por outros autores (entre 10-12\%) (Rocha et al., 2008; Pereira et al., 2012) e diferindo de outros autores, onde a maioria foi de cães de raça pura (Pereira et al., 2012; Pereira et al., 2015). Esse fato pode estar correlacionado com a maior frequência de SRD atendidos no hospital veterinário. Dentre os de raça, o Buldogue Francês, Yorkshire e Poodle foram os mais acometidos (8\% cada), sendo os dois últimos de pelagem longa diferindo da literatura que descreve que cães de pelo curto foram mais observados (Pereira et al., 2012).

A maior casuística de cães com a forma generalizada (Pereira et al., 2012; Pereira et al., 2015) está de acordo com o encontrado, porém difere de outro estudo onde a localizada foi maior
(Nayak et al., 1997). A localizada tem curso benigno e a maioria dos casos tem resolução espontânea (Miller et al., 2013), talvez por esse motivo o maior número de atendimentos de animais com a forma generalizada que normalmente se inicia quando ainda filhotes (3-18 meses) (Miller et al., 2013)

Observou-se que a região da cabeça foi a mais acometida, assim como reportado por Pereira et al. (2012), e mesmo nos casos generalizados, a cabeça também é descrita como o local de maior ocorrência (Delayte, 2016). Uralet al. (2012) obtiveram ácaros na pele dos membros, cabeça e tronco e o envolvimento principalmente das orelhas, olhos, lábios, queixo e pernas. As lesões frequentemente começam na face e nas patas dianteiras, podendo explicar o maior acometimento dessas áreas (Mueller et al., 2012). Lesões acometendo todo o corpo aconteceram em 30,4\% dos casos.

Alopecia focal, eritema e descamação estão entre os sinais clínicos mais frequentes (Mueller, 2012) e no presente estudo, hipotricose, eritema e crosta, porém alopecia e descamação também apresentaram alta ocorrência com $42,4 \%$ cada. Ulceração focal e tratos drenantes estão correlacionadas a casos mais avançados (Mueller et al., 2012) e foram menos observados neste estudo. Pústulas, colaretes, crostas e fístulas também observadas estão correlacionadas com a presença de infecção bacteriana secundária (Delayte, 2016).

Dentre as técnicas utilizadas para o diagnóstico, o raspado cutâneo foi mais utilizado, corroborando com a literatura como método diagnóstico padrão (Saridomichelakis et al., 2007; Delayte, 2016). A impressão em fita de acetato também foi bastante utilizada e é menos traumática e dolorosa, sendo útil em áreas difíceis de obter amostras (Pereira et al., 2012). O tricograma, considerada uma técnica menos sensível para o diagnóstico (Cury et al., 2013), foi realizado em apenas $21,6 \%$,

O exame citológico foi utilizado em 17,6\% dos cães e é indicado em casos com lesões exsudativas de pústulas e tratos drenantes (Mueller et al., 2012) que, menos frequentes nesse estudo, e talvez por isso a menor observação de casos com ácaros através desta técnica. Já a biópsia cutânea, método diagnóstico menos utilizado nesse estudo, é mais indicada em casos que não foi possível observar o ácaro em exames diretos (Mueller et al., 2012; Delayte,2016), como em cães da raça Sharpei, com a pele mais espessa (Mueller, 2012) que 
corresponderam a apenas $2,4 \%$ dos casos e, como o raspado tem alta sensibilidade (Saridomichelaki set al., 2007), a necessidade de biópsia cutânea foi menor.

Vale ressaltar que diferentes técnicas foram realizadas em um mesmo animal. Não foi objetivo do estudo a comparação das técnicas, avaliando sua sensibilidade e especificidade e, sim, reportar a ocorrência da doença nos cães.

\section{Conclusão}

Foi possível concluir que a demodiciose é uma doença que deve estar dentre os diagnósticos diferenciais na clínica de animais de companhia, principalmente em animais jovens, com idade inferior a 1 ano de idade. $\mathrm{E}$ que esta doença acomete principalmente região de cabeça com lesões mais frequentes de hipotricose e eritema.

\section{Conflito de Interesse}

Os autores declaram não existir conflito de interesse.

\section{Referências}

Bowden, D.; Outerbridge, C.; Kissel, M.B.; Baron, J.N.; White, S.D. Canine demodicosis: a retrospective study of a veterinary hospital population in California, USA (2000-2016). Veterinary Dermatology, 28:1-9, 2017.

Cury, G.M.M.; Pereira, S.T.; Botoni, L.S.; Pereira, R.D.O.; Telles, T.C.; Ferreira, A.P.; CostaVal, A.P. Diagnosis of canine demodicosis: comparative study between hair pluck in gand adhesive tape tests. Revista Brasileira de Ciência Veterinária, 20 (3): 137-139, 2013.

Delayte, E.H.; Otsuka, M.; Larsson, C.E.; Castro, R.C.C. Eficácia das lactonas macrocíclicas sistêmicas (ivermectina e moxidectina) na terapia da demodicidose canina generalizada. Arquivo Brasileiro de Medicina Veterinária e Zootecnia, 58(1):31-38, 2006

Delayte, E.H. Demodiciose canina. In: Larsson, C.E.; Lucas, R. Tratado de medicina externa- dermatologia veterinária. $1^{\mathrm{a}} \mathrm{ed}$. São Caetano do Sul: Interbook, 2016, p.369389.

Ferrer, L.; Ravera, I.; Silbermayr, K. Immunology and pathogenesis of canine demodicosis. Veterinary Dermatology, 25:427-e65, 2014.

Gasparetto, N.D.; Trevisan, Y.P.A.; Almeida, N.B.; Neves, R.C.S.M.; Almeida, A.B.P.F.; Dutra, V.; Colodel, E.M.; Sousa, V.R.F. Prevalência das doenças de pele não neoplásicas em cães no município de Cuiabá, Mato Grosso. Pesquisa Veterinária Brasileira, 33 (3): p.359-362, 2013.

Ghubash, R. Parasitic miticidal therapy. Clinical Techniques in Small Animal Practice, 21(3):135-44, 2006.

Holm, B. Efficacy of milbemycinoxime in the treatment of canine generalized demodicosis: a retrospective study of 99 dogs (1995-2000). Veterinary Dermatology, 14:189-195, 2003.

Lemarie, S.L.; Hosgood, G.; Foil, C.S. A retrospective study of juvenile- and adultonset generalized demodicosis in dogs (198691). Veterinary Dermatology, 7: p.3-10, 1996.

Miller, W.H.; Griffin, C.E.; Campbell, K.L. Muller and Kirk's small animal dermatology. $7^{\text {th }}$ ed. St Louis: Elsevier, 2013, 948p.

Mueller, R. S.; Bensignor, E.; Ferrer, L.; Holm, B.; Lemarie, S.; Paradis, M.; Shipstone, M. A. Treatment of demodicosis in dogs: 2011 clinical practice guidelines, Veterinary Dermatology, 23:86-e21, 2012.

Nayak, D.C.; Tripathy, S.B.; Dey, P.C.; Ray, S.K.; Mohanty, D.N.; Parida, G.S.; Biswal, S.; DAS, M. Prevalence of canine demodicosis in Orissa (India). Veterinary Parasitology, 73:347-352, 1997.

Plant, J.D.; Lund, E.M.; Yang, M. A case-control study of the risk factors for canine juvenileonset generalized demodicosis in the USA. Veterinary Dermatology, 22: 95-99, 2010.

Pereira, A.V.; Pereira, S.A.; Gremião, I.D.F.; Campos, M.P.; Ferreira, A.M.R. Comparison of acetate tape impression with queezing versus skin scraping for the diagnosis of canine demodicosis. Australian Veterinary Journal, 90(11):448-450, 2012.

Pereira, D.T.; Castro, L.J.M.; Centenaro, V.B; Amaral, A.S; Krause, A.; Schmidt, C. Skin impression with acetate tape in Demodex canis and Scarcoptes scabiei var. vulpes diagnosis. Arquivo Brasileiro de Medicina Veteterinária e Zootecnia, 67(1):49-54, 2015

Ravera, I.; Altet, L.; Francino, O.; Sánchez, A.; Rolda, N.W.; Villanueva, S.; Bardagi, M.; Ferrer, L. Small Demodex populations colonize most parts of the skin of healthy dogs. Veterinary Dermatology, 24:168-e37, 2013. 
Reddy, B.S.; Sivajothi, S. CD4+ and CD8+ T cells in the peripheral blood of dogs affected with generalized demodicosis. Comparative Clinical Pathology, 25:295-297, 2016.

Rocha, G.S.; Ahid, S.M.M.; Bezerra, A.C.D.S.; Filgueira, K.D.; Santos, J.P.S.S. Frequiência de ácaros em cães e gatos no município de Mossoró, Rio Grande do Norte. Acta Scientiae Veterinariae, 36(3): 263-266, 2008.

Saridomichelakis, M.N.; Koutinas, A.F.; Farmaki, R.; Leontides, L.S.; Kasabalis, D. Relative sensitivity of hair pluckings and exudate microscopy for the diagnosis of canine demodicosis. Veterinary Dermatology, 18 (2):138-41, 2007.

Silva, L.V.A.; Santana, I.M.; Alves, L.C.; Faustino, M.A.G. Infecção por Demodex canis em cães dermatologicamente sadios e com dermatopatias, procedentes da região metropolitana de Recife, Estado de Pernambuco. Medicina Veterinária, 5 (3):16, 2011.

Sivajothi, S.; Reddy, B.S.; Rayulu, V.C. Demodicosis caused by Demodex canis and Demodex cornei in dogs. Journal of Parasitic Diseases, 39 (4):673-676, 2015.

Ural, K.; Voyvoda, H.; Ulutaş, B.; Paşa, S.; Aysul, N.; Gültekin, M. Understanding Primary and Secondary Skin Lesions among Infectious Dermatoses in Dogs: Lessons We Learned From Cases. Animal Health, Production and Hygiene, 1(2):86-99, 2012. 